\title{
Evidence of Quantum Resonance in Periodically-Ordered Three- Dimensional Superlattice of CdTe Quantum Dots
}

\section{AUTHOR(S):}

Kim, DaeGwi; Tomita, Shougo; Ohshiro, Kazuma; Watanabe, Taichi; Sakai, Takenobu; Chang, I-Ya; Kim, Hyeon-Deuk

\section{CITATION:}

Kim, DaeGwi ...[et al]. Evidence of Quantum Resonance in Periodically-Ordered ThreeDimensional Superlattice of CdTe Quantum Dots. Nano Letters 2015, 15(7): 4343-4347

\section{ISSUE DATE:}

2015-07-08

URL:

http://hdl.handle.net/2433/229438

\section{RIGHT:}

This document is the Accepted Manuscript version of a Published Work that appeared in final form in 'Nano Letters', copyright ( $)$ American Chemical Society after peer review and technical editing by the publisher. To access the final edited and published work see https://doi.org/10.1021/acs.nanolett.5b00335.; The full-text file will be made open to the public on 19 June 2016 in accordance with publisher's 'Terms and Conditions for Self-Archiving'. This is not the published version. Please cite only the published version.この論文は出版社版でありません。引用の際には出版社版を ご確認ご利用ください。 


\section{Evidence of Quantum Resonance in Periodically-}

\section{Ordered Three-Dimensional Superlattice of CdTe}

\section{Quantum Dots}

DaeGwi Kim* $^{*}$, , Shougo Tomita ${ }^{\dagger}$, Kazuma Ohshiro ${ }^{\dagger}$, Taichi Watanabe ${ }^{\dagger}$, Takenobu Sakai ${ }^{\ddagger}$, I-Ya Chang ${ }^{\S, \|}$ and Kim Hyeon-Deuk*,§,

${ }^{\dagger}$ Department of Applied Physics, Osaka City University, Osaka 558-8585, Japan

†Green Mobility Collaborative Research Center, Nagoya University, Nagoya 464-8603, Japan

${ }^{\S}$ Department of Chemistry, Kyoto University, Kyoto 606-8502, Japan

"Japan Science and Technology Agency, PRESTO, 4-1-8 Honcho, Kawaguchi, Saitama 3320012, Japan

\section{Corresponding Author:}

*E-mail: : tegi@a-phys.eng.osaka-cu.ac.jp; kim@kuchem.kyoto-u.ac.jp 
ABSTRACT: Semiconductor quantum dot (QD) superlattices, which are periodically ordered three dimensional (3D) array structures of QDs, are expected to exhibit novel photo-optical properties arising from the resonant interactions between adjacent QDs. Since the resonant interactions such as long-range dipole-dipole Coulomb coupling and short-range quantum resonance strongly depend on inter-QD nano space, precise control of the nano space is essential for physical understanding of the superlattice which includes both of nano and bulk scales. Here, we study the pure quantum resonance in the 3D CdTe QD superlattice deposited by a layer-bylayer assembly of positively charged polyelectrolytes and negatively charged CdTe QDs. From XRD measurements, existence of the periodical ordering of QDs both in the lamination and inplane directions, that is, the formation of the 3D periodic QD superlattice was confirmed. The lowest excitation energy decreases exponentially with decreasing the nano space between the CdTe QD layers and also with decreasing the QD size, which is apparently indicative of the quantum resonance between the QDs rather than a dipole-dipole Coulomb coupling. The quantum resonance was also computationally demonstrated and rationalized by the orbital delocalization to neighboring CdTe QDs in the superlattice.

KEYWORDS: Quantum dot, Three-dimensional superlattice, Quantum resonance, HOMO/LUMO delocalization, density functional theory 
Since the first report of quantum size effects in semiconductor-doped glasses ${ }^{1}$ and also in colloidal solutions ${ }^{2}$ in the early 1980s, semiconductor QDs have attracted considerable attention to understand the size dependence of their physical and/or chemical properties due to the demand for developments of novel optoelectronic and photovoltaic devices. While randomly dispersed QDs have been a major target in most of studies, in hyper-structure QD materials, such as twodimensional (2D) QD films and three-dimensional (3D) QD superlattices, realization of novel optical properties and functions based on quantum interactions between adjacent QDs have been expected $^{3-5}$. Such interactions are the so-called resonant coupling including the two typical interactions between adjacent QDs, a long-range dipole-dipole Coulomb coupling and shortrange quantum resonance.

The studies about the resonant coupling between semiconductor QDs have attracted much attention quite recently due to difficulty of the sample preparation for realizing the resonant coupling. The resonant coupling has been discussed in the close-packed 2D films composed of QDs such as $\mathrm{InP}^{6}, \mathrm{CdS}^{7}$, and $\mathrm{CdSe}^{8}$ so far. The absorption cross section is enhanced by 4-5 times due to the dipole-dipole couplings ${ }^{9}$, while the absorption peaks of the close-packed QD films are shifted toward lower energies compared with the peaks of the corresponding dilute solution samples where each QD is almost isolated ${ }^{10}$. The red shift of the absorption peak implies the quantum resonance between neighboring QDs. However, the disordered 2D QD film structures inevitably involve various kinds of the resonant couplings like the long-range dipole-dipole coupling, making it difficult to elucidate an evidence of the pure quantum resonance ${ }^{4,5,11}$. Since the resonant coupling strongly depends on nano space between neighboring QDs, precise and 
systematic manipulation of the nano space is indispensable for controlling the resonant coupling and for purely deducing the short-range quantum resonance.

For this purpose, we have focused on a layer-by-layer (LBL) assembly. The LBL assembly process is a simple and convenient method for preparing highly homogeneous QD/polymer multilayers as pointed out by Decher and co-workers ${ }^{12,13}$ and also by Kotov ${ }^{14}$. An important feature of the LBL assembly from the viewpoint of the resonant coupling is that the nano space between homogeneous QD monolayers can be precisely controlled by the spacer-layer thickness with nanometer accuracy by using the LBL assembly of oppositely charged polyelectrolytes ${ }^{15}$.

As schematically shown in Fig. 1a, we carefully prepared CdTe QD multilayers by the LBL assembly of positively charged polyelectrolytes of poly (diallyldimethylammonium chloride) (PDDA) and negatively charged CdTe QDs, (PDDA/CdTe-QDs) $n$. Here, the notation of $(\mathrm{PDDA} / \mathrm{CdTe}-\mathrm{QDs})_{n}$ represents an $n$-layer structure composed of PDDA and CdTe QDs. Figure $1 \mathrm{~b}$ and 1c show out-of plane and in-plane XRD patterns of the current CdTe QD multilayer (PDDA/CdTe-QDs) 8 , respectively. The observation of the diffraction peaks at $2.51^{\circ}$ in Fig. $1 \mathrm{~b}$ and at $1.90^{\circ}$ in Fig. 1c indicates that the current CdTe QD multilayers have a structural regularity with a periodicity of $\sim 3.5 \mathrm{~nm}$ in the lamination direction and of $\sim 4.6 \mathrm{~nm}$ in the in-planed direction. The existence of these periodic structural ordering of CdTe QDs not only in the lamination direction but also in the in-plane direction clearly demonstrates the formation of the 3D periodic CdTe QD superlattices. The successful preparation of the 3D periodic QD superlattice enables us to independently observe the quantum resonance between periodically aligned CdTe QDs. 
Figure 2a shows absorption spectra of (PDDA/CdTe-QDs) ${ }_{n}$ for a different number of layers $n$. The absorption intensity monotonically increases with the layer number $n$. The dependence of the optical density at the absorption peak is also shown in Fig. 2b. The linear dependence of the optical density on the layer number is clearly observed, indicating that the CdTe QDs are adsorbed uniformly layer by layer during the LBL deposition in proportion to the layer number. This is an advantage of the LBL assembly for preparing a homogeneous 3D QD superlattice. Figure $2 \mathrm{c}$ shows the dependence of the absorption peak energy on the layer number $n$. A noteworthy observation is that the absorption peak energy gradually shifts to lower energy with an increase in the layer number. As will be demonstrated experimentally in Fig.3 and computationally in Fig. 4, the decrease in the peak energy originates from the quantum resonance between the CdTe QD layers which mutually interact. The gradual peak shift proportional to the layer number reflects the successful LBL deposition and thus indicates the gradual formation of the homogeneous 3D superlattice. The saturated peak energy corresponds to the absorption peak energy of the 3D periodic CdTe QD superlattice. We define the difference between the absorption peak energies of the monolayer and of the 3D periodic superlattice of CdTe QDs as a total energy shift.

As discussed in ref. 3, to understand the origin of the total energy shift appearing in the CdTe QD superlattice, we need to consider physical difference between the dipole-dipole Coulomb coupling and the quantum resonance. The former arises from a Coulomb interaction among QDs, while the latter stems from overlap of wave functions between adjacent QDs. Therefore, the dipole-dipole Coulomb coupling is long-range interaction, and its strength should be inversely proportional to the 6-th power of the separation distance ${ }^{9,16}$. On the other hand, the quantum 
resonance is quite short-range, and its magnitude should depend exponentially on the distance between $\mathrm{QDs}^{3}$.

To distinguish these two interactions, it is indispensable to measure dependence of the energy shift on the nano space between the CdTe QD layers. The nano space between the bilayer structure, CdTe-QDs/(PDDA/PAA)m/PDDA/CdTe-QDs can be systematically the spacer $(\mathrm{PDDA} / \mathrm{PAA})_{m}$. Here, $(\mathrm{PDDA} / \mathrm{PAA})_{m}$ represents the $m$ layers composed of positively charged PDDA and negatively charged polyacrylic acid (PAA), which are deposited by the LBL assembly. The average thickness of the single PDDA/PAA layer is estimated to be $0.9 \mathrm{~nm}$ from the spectroscopic ellipsometry measurement ${ }^{15}$. Thus, the nano space between the CdTe QD layers can be systematically controlled with the accuracy of $0.9 \mathrm{~nm}$.

Figure 3 a shows the dependence of the energy shift between the absorption peaks of the CdTe QD monolayer and of the CdTe QD bilayer on the layer number $m$ of (PDDA/PAA $)_{m}$. As shown in the figure, the energy shift decreases exponentially with the nano space between the layers of the CdTe QD bilayer. This exponential decay clearly demonstrates that the current interaction through the nano space is not the long-range dipole-dipole Coulomb coupling but rather the quantum resonance between the adjacent CdTe QDs in the lamination direction. This conclusion is further supported by Fig. $3 b$.

Figure $3 \mathrm{~b}$ shows the dependence of the total energy shift of the absorption peak for the 3D CdTe QD superlattice from the absorption peak of the CdTe QD monolayer as a function of the mean diameter of the CdTe QDs. The solid curve denotes an exponential fitting function which well explains the experimental results. The total energy shift exponentially decreases with increasing the QD diameter, reflecting that the quantum resonance becomes weaker 
exponentially with larger QD diameter. As will be computationally demonstrated in Fig. 4, overlap of wave functions of neighboring CdTe QDs causes the current quantum resonance. With a decrease in the QD diameter, the penetration of the wave function outside the QD caused by the interaction from the neighboring QDs becomes larger due to the larger surface ratio over the volume, which results in the stronger quantum resonance. The current exponential decrease of the total energy shift can be attributed to the physical expectation that the wave function overlap decreases exponentially with increasing QD diameter, and can be clear evidence of the quantum resonance caused in the 3D periodic QD superlattice. Here, we note that the long-range dipole-dipole coupling does not play a role in the current superlattice which possesses the small and well-organized nano space between QDs since the overlap of the wave functions to induce the quantum resonance itself prevents a formation of a dipole at the center of each QD. The diameter of the QDs also affects the saturation distance $(A, A, B)$ as well as the total energy shift. In fact, the total energy shift almost vanishes in the QDC composed of $4.5 \mathrm{~nm}$ QDs, indicating that the QD resonance almost disappears at the given nano space. The QDCs composed of smaller QDs still exhibit the finite total energy shift, demonstrating that a smaller QD elongates the saturation distance due its larger quantum resonance.

In order to rationalize the quantum resonance implied in the experimental data, we perform density functional theory calculations on a 3D CdTe QD superlattice composed of the $\mathrm{Cd}_{26} \mathrm{Te}_{26}$ QD whose diameter is approximately $1.5 \mathrm{~nm}$. The CdTe QD superlattice is made by periodically replicating the cubic cells in $x y z$ dimensions. We put nano space of $A$ between nearest-neighbor QD surfaces in each $x y$-layer and set nano space of $B$ between the layers along the $z$-axis. The CdTe QD superlattice geometry is thus characterized by the 3D nano space between the QDs, $(A$, $A, B)$ as graphically shown in the insert of Fig. 4a. 
Figure 4a shows energy shifts of the HOMO-LUMO band gap depending on the nano space $(A, A, B)$. The band gap energy decreases with the smaller $(A, A, B)$ nano space, which agrees with the experimental observation in Fig. 3a. Such shifts are recovered with increasing the nano space $(A, A, B)$, and the band gap energy finally saturates at around $(A, A, B)=(1.0 \mathrm{~nm}, 1.0 \mathrm{~nm}$, $1.0 \mathrm{~nm}$ ). The saturated energy corresponds to the band gap energy of the isolated $\mathrm{Cd}_{26} \mathrm{Te}_{26} \mathrm{QD}$, $1.183 \mathrm{eV}$, calculated at $(A, A, B)=(3.0 \mathrm{~nm}, 3.0 \mathrm{~nm}, 3.0 \mathrm{~nm})$. If we set all the saturated energies at large $B$ for every $A$ as $1.183 \mathrm{eV}$, all the shift profiles are almost on the universal exponential line, which is again in harmony with the experimental data.

The shifts of the band gap energy are closely related to and rationalized by the quantum resonance between nearest-neighbor QDs in the superlattice; the quantum resonance caused at the small nano space reduces the band gap energies, while the energy shifts are recovered as the quantum resonance becomes weaker at the larger nano space. Figure $4 b$, which shows LUMO at various nano space, supports this scenario; the LUMO on the $(A, A)$-plane is delocalized with $A$ of $0.3 \mathrm{~nm}$ as shown in the elongated orbital along the horizontal plane, while it becomes localized with $A$ of $1.5 \mathrm{~nm}$. The LUMO along $B$-axis is delocalized with $B$ of $0.4 \mathrm{~nm}$ as shown in the elongated orbital along the vertical axis, while it becomes localized with $B$ of $0.8 \mathrm{~nm}$. The delocalized LUMO over the neighboring QDs indicates the strong quantum resonance at the small nano space. The nano space range where the LUMO is delocalized is coincident with the threshold nano space for the shifts of the band gap energy estimated above. We note that the LUMO plays a more important role in the quantum resonance than the HOMO as compared in Fig. S1. The delocalization of the LUMO indicates an ultrafast electron transfer and an effective charge separation in the 3D QD superlattice. 
The experimental threshold corresponding to the nano space $B$ is longer than the current threshold because positive charges in the PDDA polymers existing between the CdTe QD layers can enhance the quantum resonance. This conclusion that the current CdTe QD superlattice with vacuum nano space $(A, A, B)$ results in the shorter nano space threshold suggests that the quantum resonance and thus the energy shift can be manipulated by changing a charge and dielectric property of the nano space. In addition, the current larger energy shift compared to the total energy shift experimentally observed in Fig. 3a can be rationalized by extrapolating the exponential dependence of the total energy shift on the QD diameter shown in Fig. 3b. The charged nano space in the experimental CdTe QD superlattice also influences the amplitude of the energy shift as well as the nano space threshold.

Figure $4 \mathrm{c}$ represents the density of states (DOS) of the VB and CB. Although the band gap energy becomes larger as $A$ and $B$ increases,, the band edge energies in the VB and $\mathrm{CB}$ are all set to zero in Fig. 4c to directly compare the VB and CB DOS at the different nano space. The whole shapes of the CB and VB DOS are maintained regardless of the nano space. However, the DOS at the small nano space expressed by the red line is totally distorted toward the higher energy. Such DOS distortion as well as the band gap shifts reported in Fig. 4a are attributed to the deformation of $\mathrm{Cd}_{26} \mathrm{Te}_{26} \mathrm{QD}$ in the superlattice. Figure $4 \mathrm{~d}$ graphically compares the $\mathrm{Cd}_{26} \mathrm{Te}_{26}$ QD at the small and large nano space. With the small nano space, each $\mathrm{Cd}_{26} \mathrm{Te}_{26} \mathrm{QD}$ is compressed by the inter-QD interaction from the adjacent QDs. This fact can be confirmed by the shrunk $\mathrm{Cd}_{26} \mathrm{Te}_{26} \mathrm{QD}$ structures at $(A, A, B)=(0.2 \mathrm{~nm}, 0.2 \mathrm{~nm}, 0.1 \mathrm{~nm})$ compared to the structure at $(A, A, B)=(1.5 \mathrm{~nm}, 1.5 \mathrm{~nm}, 2.0 \mathrm{~nm})$. The estimated root mean square displacement (RMSD) for the deviation between the former and latter $\mathrm{Cd}_{26} \mathrm{Te}_{26}$ QDs is $0.0591 \mathrm{~nm}^{17}$. The 
mathematical definition of RMSD is given by $\sqrt{\frac{1}{N} \sum_{i<j}^{N}\left(\rho_{i}-\rho_{j}\right)^{2}}$ where $N$ is the number of atoms constituting a QD and $r_{i}$ is a vector of Cartesian coordinates of $i$-th atom. The other structural comparisons of the $\mathrm{Cd}_{26} \mathrm{Te}_{26} \mathrm{QD}$ in the superlattice from the different views are given in Fig. S2. The current structural deformation is caused by strong Coulomb interactions between positive Cd and negative Te atoms through the small nano space; the QD is compressed due to the repulsive force between same kind of atoms, $\mathrm{Cd}$ and $\mathrm{Cd}$ or Te and Te, of the neighboring QDs, while the QD can be expanded due to the attractive force between differently charged atoms, Cd and Te, of the adjacent QDs. In the current case, the repulsive force between the same kind of atoms has a stronger effect on the deformation of the QD rather than the attractive force between $\mathrm{Cd}$ and $\mathrm{Te}$, leading to the slight compression of the QD. Actually, the QD diameters along the xyz-directions are $(1.39 \mathrm{~nm}, 1.38 \mathrm{~nm}, 1.16 \mathrm{~nm})$ and $(1.40 \mathrm{~nm}, 1.40 \mathrm{~nm}, 1.24 \mathrm{~nm})$ for the nano space set as $(A, A, B)=(0.2 \mathrm{~nm}, 0.2 \mathrm{~nm}, 0.1 \mathrm{~nm})$ and $(1.5 \mathrm{~nm}, 1.5 \mathrm{~nm}, 2.0 \mathrm{~nm})$, respectively. In fact, if we did not optimize each $\mathrm{Cd}_{26} \mathrm{Te}_{26}$ QD in the unit cell of the QD superlattice, significantly different results would be obtained; the total energy of the QD superlattice for $(A, A, B)=(0.2 \mathrm{~nm}, 0.2 \mathrm{~nm}, 0.1 \mathrm{~nm})$ with the relaxed structure of the isolated QD is $-103.07 \mathrm{eV}$, while the total energy of the QD superlattice with the current deformed QD is $-117.68 \mathrm{eV}$, indicating that the QD superlattice composed of the current deformed QDs is more stable. Further, we optimized the QD superlattice with a unit cell composed of a QD dimer, that is, two $\mathrm{Cd}_{26} \mathrm{Te}_{26}$ QDs. As shown in Fig.S5, the obtained QD geometries, LUMO and band gap shifts are all close to the results with the single $\mathrm{Cd}_{26} \mathrm{Te}_{26}$ QD unit cell reported here, demonstrating that the current single $\mathrm{Cd}_{26} \mathrm{Te}_{26}$ QD unit cell is competent to compute the periodic properties of the CdTe QD superlattice. 
In this work, the pure quantum resonance in the 3D periodic QD superlattice was for the first time found and rationalized both experimentally and theoretically by systematically controlling the nano space between QDs. We believe that the present results and findings have a major impact on how to design and synthesize hyper-structured materials composed of nanomaterials with nano space, and provide a new insight into their photovoltaic properties which the disordered 2D QD films nor the 3D superlattices with the long-range inter-QD distance do not exhibit.

\section{References}

(1) Ekimov, A. I.; Onushchenko, A. A. Sov. Phys. Semiconductors-USSR 1982, 16, 775.

(2) Henglein, A. Ber. Bunsenges. Phys. Chem. 1982, 86, 301.

(3) Hanrath, T. J. Vac. Sci. Technol. A 2012, 30, 030802.

(4) Choi, J.-H.; Fafarman, A . T.; Oh, S. J.; Ko, D.-K.; Kim, D. K.: Diroll, B. T.; Muramoto, S.; Gillen, J. G.; Murray, C. B.; Kagan, C. R. Nano Lett. 2012, 12, 2631.

(5) Talgorn, E.; Gao, Y.; Aerts, M.; Kunneman, L. T.; Schins, J. M.; Savenije, T. J.; Herre, M. A. H.; Zant, S. J.; Houtepen, A. J.; Siebbeles, L. D. A. Nature Nanotech. 2011, 6, 733.

(6) Mićić, O. I.; Ahrenkiel, S. P.; Nozik, A. J. Appl. Phys. Lett. 2001, 78, 4022.

(7) Döllefeld, H.; Weller, H.; Eychmüller, A. J. Phys. Chem. B 2002, 106, 5604.

(8) Artemyev, M. V.; Woggon U.; Jaschinski, H.; Gurinovich, L. I.; Gaponenko, S. V. J. Phys. Chem. B 2000, 104, 11617. 
(9) Geiregat, P.; Justo, Y.; Abe, S.; Flamee, S.; Hens Z. Nano Lett. 2013, 7, 987.

(10) Hodes, G.; Grunbaum, E.; Feldman, Y.; Bastide, S.; Lévy-Clément, C. J. Elec. Soc. 2005, 152, G917.

(11) Lee, J.-S.; Kovalenko, M. V.; Huang, J.; Chung, D. S.; Talapin, D. V. Nature Nanotech. 2011, 6, 348 .

(12) Decher, G. Science 1997, 277, 1232.

(13) Decher, G.; Schlenoff, J. B. "Multilayer Thin Films" (Wiley-VCH 2003).

(14) Kotov, N. A. “Nanoparticle Assemblies and Superstructures” (CRC Press 2005).

(15) Kim, D.; Okahara, S.; Nakayama, M.; Shim, Y. Phys. Rev. B 2008, 78, 153301.

(16) Yang, Y.; Liu, Z.; Lian, T. Nano Lett. 2013, 13, 3678.

(17) (18) Bu, H.; Kikunaga, H.; Shimura, K.; Takahashi, K.; Taniguchi, T.; Kim, D. Phys. Chem. Chem. Phys. 2013, 15, 2903.

(19) Kresse, G.; Furthmüller, J. Phys. Rev. B 1996, 54, 11169.

(20) Perdew, J. P.; Burke, K.; Ernzerhof, M. Phys. Rev. Lett. 1996, 77, 3865.

(21) Kresse, G.; Joubert, D. Phys. Rev. B 1999, 59, 1758.

(22) Weigend, F.; Ahlrichs, R. Phys. Chem. Chem. Phys. 2005, 7, 3297.

(23) Gaussian 09, Revision A.2, M. J. Frisch et al. Gaussian, Inc., Wallingford CT, (2009). 


\section{FIGURES}

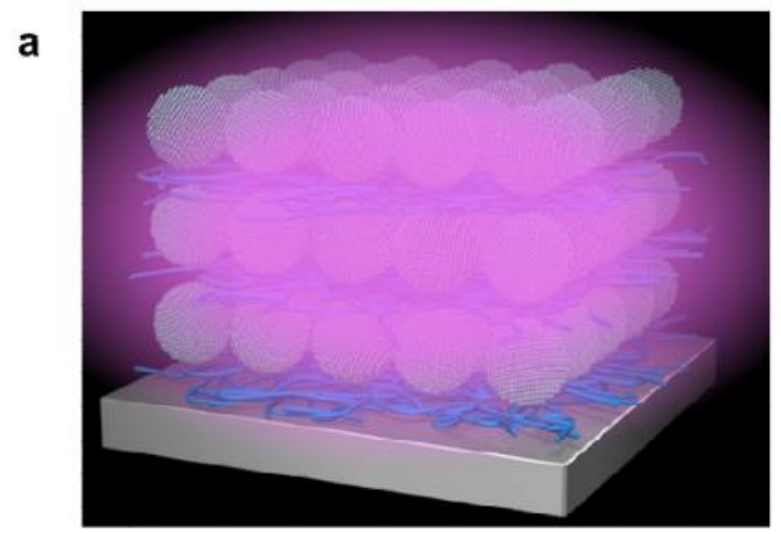

b

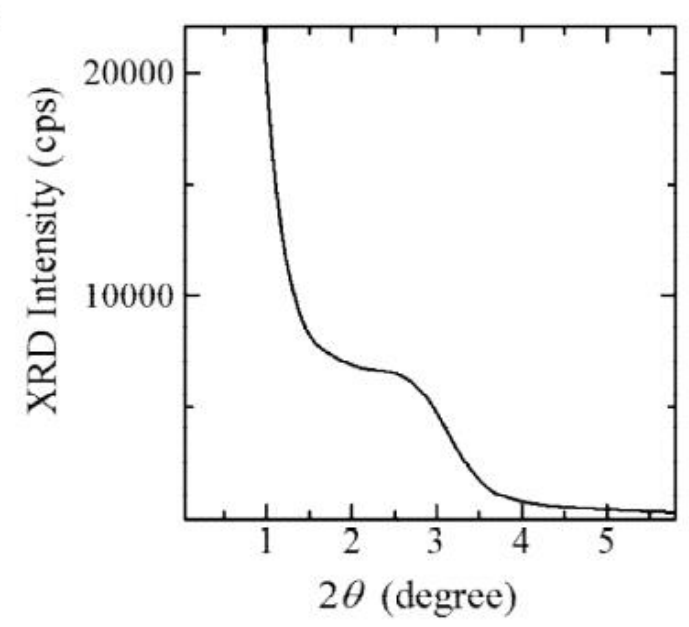

c

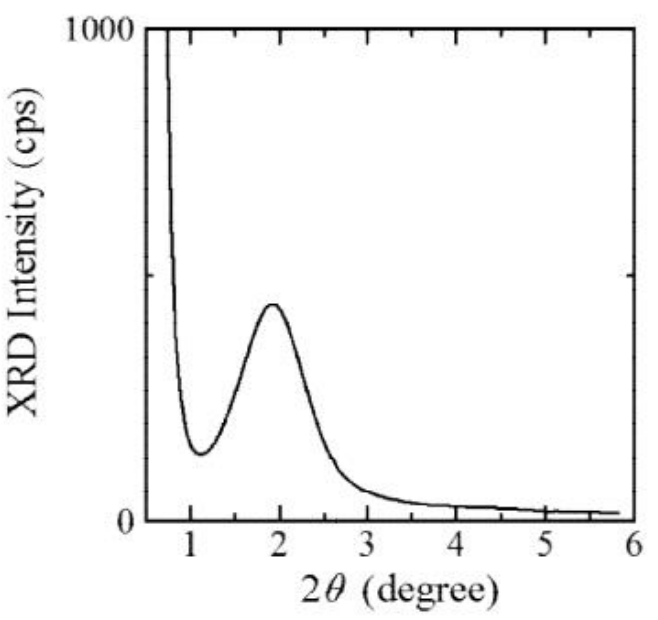

Figure 1. (a) Sketch of the multilayer structure of CdTe QDs deposited by a LBL assembly of positively charged PDDA and negatively charged CdTe QDs. (b), (c) Results of XRD measurements. The diffraction peaks both in the out-of plane (b) and in-plane (c) XRD profiles 
demonstrate the existence of the periodic ordering of QDs not only in the lamination direction but also in the in-plane direction, that is, the formation of the CdTe QD superlattice.

a

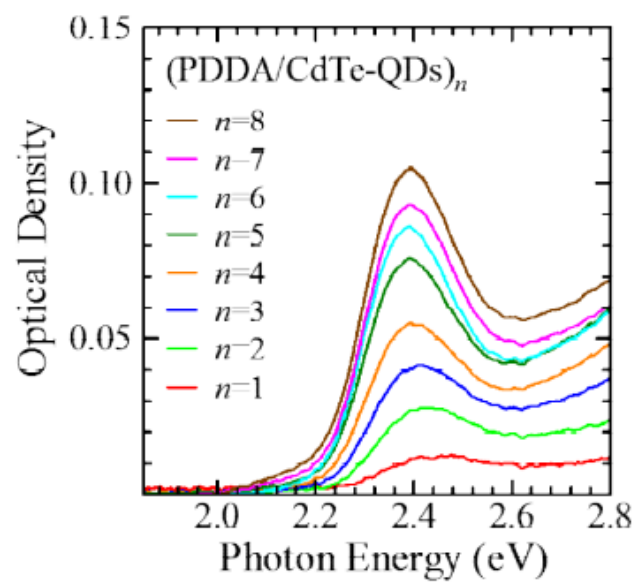

b

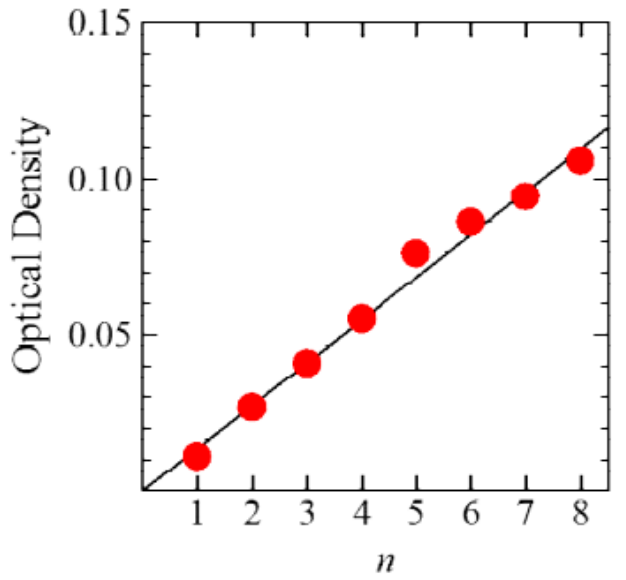

c

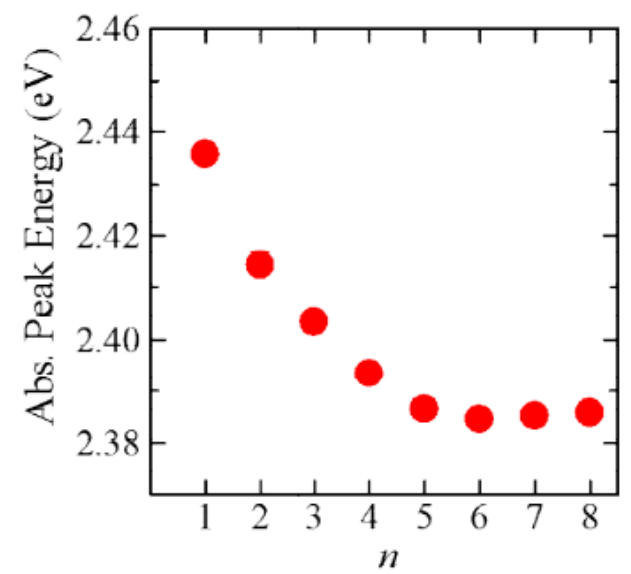

Figure 2. (a) Absorption spectra of the $n$-layer structure of CdTe QDs of (PDDA/CdTe $)_{n}$. (b) Optical density at the absorption peak energy in the CdTe QD multilayer as a function of the layer number $n$. The linear dependence of the optical density on $n$ demonstrates that the CdTe QDs were adsorbed periodically during the LBL deposition. (c) The dependence of the 
absorption peak energy on the layer number $n$. The saturated peak energy corresponds to the absorption peak energy of the CdTe QD superlattice.

a

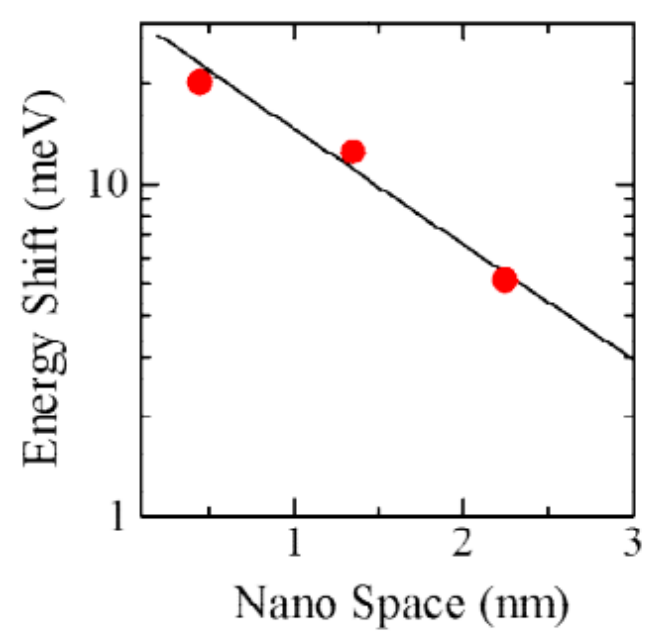

b

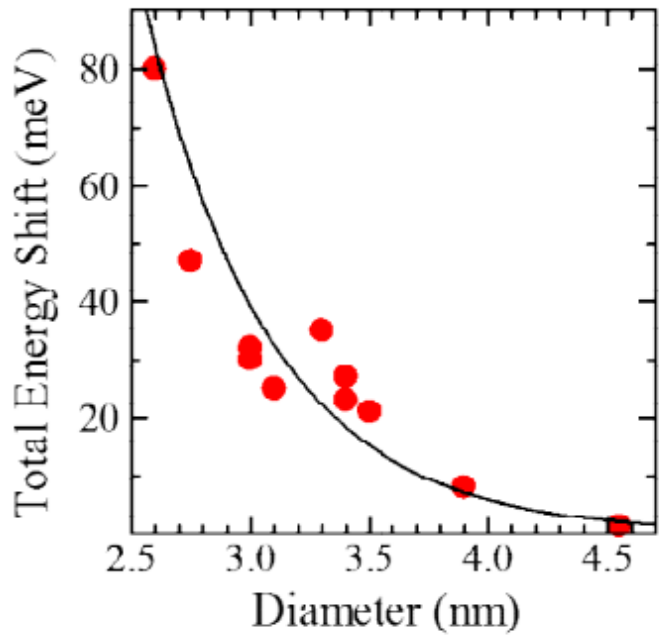

Figure 3. (a) Energy shift between the absorption peak energies of the monolayer and of the CdTe bilayer structure, CdTe-QDs/(PDDA/PAA)m/PDDA/CdTe-QDs, as a function of the layer number $m$ of (PDDA/PAA $)_{m}$. The nano space between the bilayer structure can be systematically controlled by the spacer (PDDA/PAA $)_{m}$. The exponential decrease of the energy shift with the nano space is an evidence for the short-range quantum resonance between CdTe QD layers rather than a long-range dipole-dipole Coulomb coupling between QDs. (b) Dependence of the total energy shift of the absorption peak on the mean diameter of the CdTe QDs composing the superlattice. With a decrease in the QD size, the total energy shift becomes larger, indicating the stronger quantum resonance. The solid curve represents an exponential fitting function. 


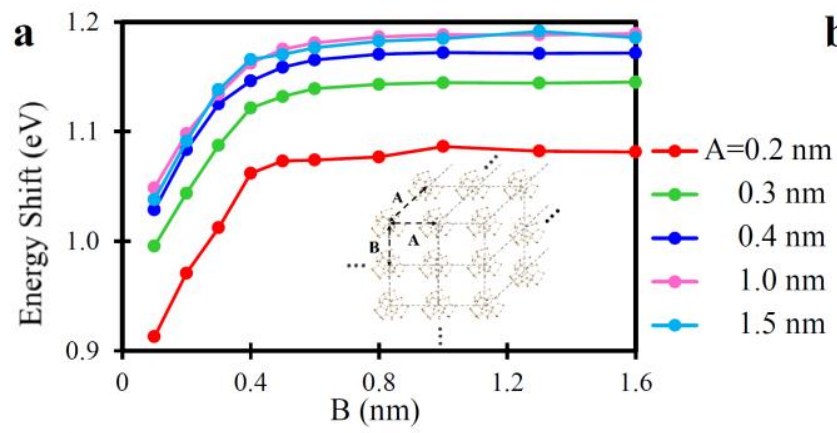

b
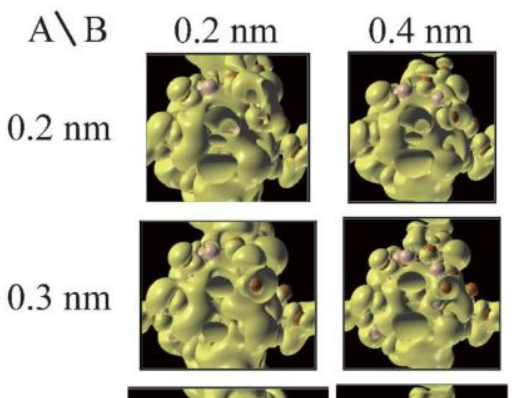

$0.8 \mathrm{~nm}$

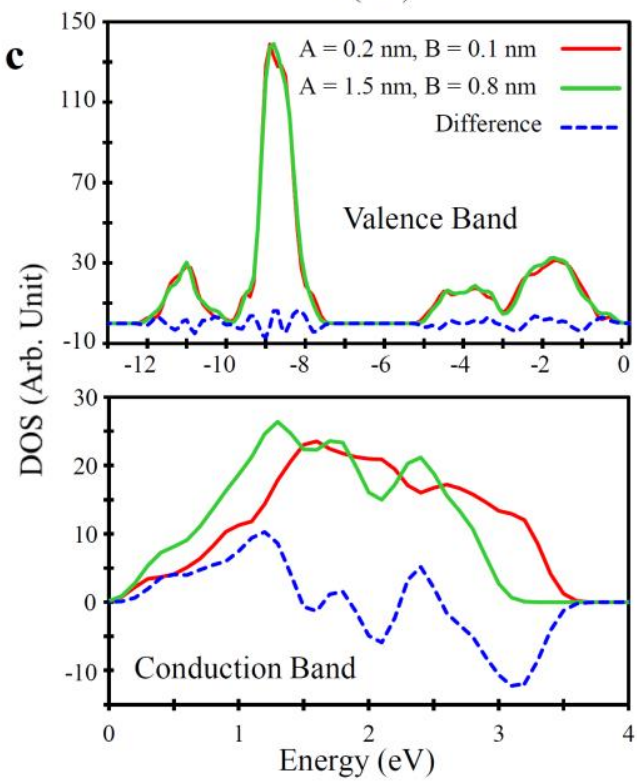

$1.5 \mathrm{~nm}$

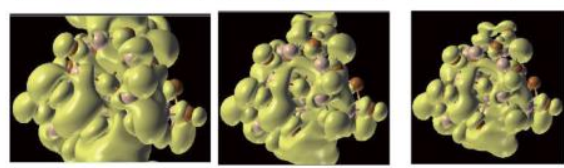

d

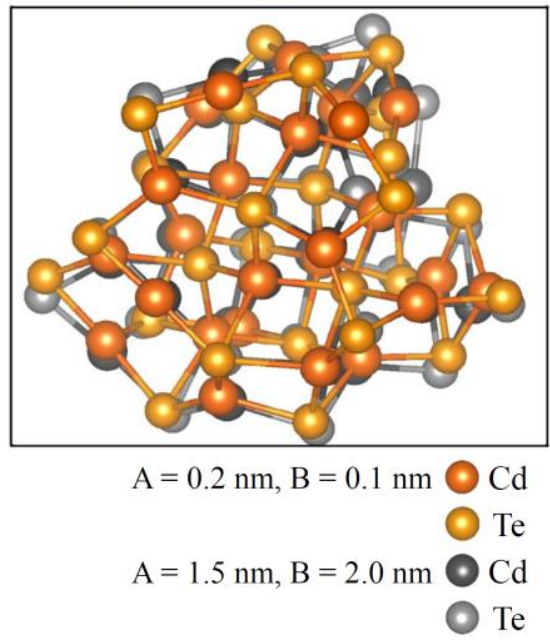

Figure 4. (a) Shifts of HOMO-LUMO band gap energies of the CdTe QD superlattice as a function of the nano space between QD surfaces in each layer, $A$, for the various nano space between the layers, $B$. The small nano space $(A, A, B)$ induces the quantum resonance between nearest-neighbor QDs, decreasing the band gap energies. The band gap energy finally saturates to the band gap energy of the isolated $\mathrm{Cd}_{26} \mathrm{Te}_{26} \mathrm{QD}$ with increasing $A$ and $B$. The maximum band gap decrease is almost one-third of the saturated band gap energy, approximately $0.3 \mathrm{eV}$. (Insert) A schematic CdTe QD superlattice structure composed of $\mathrm{Cd}_{26} \mathrm{Te}_{26}$ QDs whose diameter 
is approximately $1.5 \mathrm{~nm}$. (b) LUMO of the CdTe QD superlattice for various $A$ and $B$. The LUMO is delocalized over neighboring $\mathrm{Cd}_{26} \mathrm{Te}_{26}$ QDs with the small nano space. The delocalized LUMO becomes localized to each $\mathrm{Cd}_{26} \mathrm{Te}_{26} \mathrm{QD}$ as the nano space becomes larger. The current quantum resonance is closely related and can be rationalized by this delocalization of the LUMO in the superlattice. The delocalization of the LUMO indicates an ultrafast electron transfer and an efficient charge separation in the CdTe QD superlattice owing to the quantum resonance. (c) DOS for the CdTe QD superlattice at $(A, A, B)=(0.2 \mathrm{~nm}, 0.2 \mathrm{~nm}, 0.1 \mathrm{~nm})$ and $(1.5$ $\mathrm{nm}, 1.5 \mathrm{~nm}, 0.8 \mathrm{~nm}$ ) shown in the red and green lines, respectively. The latter corresponds to the DOS of the isolated $\mathrm{Cd}_{26} \mathrm{Te}_{26}$ QD. The whole shapes of the DOS do not significantly differ regardless of $(A, A, B)$, while, as especially seen in the $\mathrm{CB}$, the DOS at the small nano space is totally shifted to the higher energy region. (d) $\mathrm{Cd}_{26} \mathrm{Te}_{26} \mathrm{QD}$ structures in the $\mathrm{CdTe} \mathrm{QD}$ superlattice at $(A, A, B)=(0.2 \mathrm{~nm}, 0.2 \mathrm{~nm}, 0.1 \mathrm{~nm})$ and $(1.5 \mathrm{~nm}, 1.5 \mathrm{~nm}, 0.8 \mathrm{~nm})$. The former $\mathrm{Cd}_{26} \mathrm{Te}_{26} \mathrm{QD}$ is compressed due to the inter-QD interaction from the adjacent QDs, leading to the significant structural deformation. This structure change influences the band gap energy shift in Fig.4(a) as well as the DOS shift in Fig.4(c). 


\section{ASSOCIATED CONTENT}

\section{Supporting Information.}

Supporting Information for this work includes a detailed description of sample preparation, experimental methods, wide-angle X-ray diffraction pattern and absorption spectra of CdTe QDs, and orbital and geometrical structures of the $\mathrm{Cd}_{26} \mathrm{Te}_{26}$ QD in the three dimensional periodic superlattice. This material is available free of charge via the Internet at http://pubs.acs.org.

\section{ACKNOWLEDGMENT}

K. H. D. acknowledges financial support from JST (PRESTO), and Grant-in-Aids for Scientific Research from Japan Society for the Promotion of Science (KAKENHI), Grant No. 24750016. D. K. acknowledges the financial support by JSPS KAKENHI Grant Number 24560015.

\section{AUTHOR INFORMATION \\ Corresponding Author \\ *E-mail: : tegi@a-phys.eng.osaka-cu.ac.jp; kim@kuchem.kyoto-u.ac.jp}

\section{Author Contributions}

These authors contributed equally.

\section{Notes}

The authors declare no competing financial interest. 
Evidence of quantum resonance in periodically-ordered three-dimensional superlattice of CdTe quantum dot

DaeGwi Kim ${ }^{1 *}$, Shougo Tomita ${ }^{1}$, Kazuma Ohshiro ${ }^{1}$, Taichi Watanabe ${ }^{1}$, Takenobu Sakai ${ }^{2}$, I-Ya Chang ${ }^{3,4}$, Kim Hyeon-Deuk ${ }^{3,4 *}$

${ }^{1}$ Department of Applied Physics, Osaka City University, Osaka 558-8585, Japan

${ }^{2}$ Green Mobility Collaborative Research Center, Nagoya University, Nagoya 464-8603, Japan

${ }^{3}$ Department of Chemistry, Kyoto University, Kyoto 606-8502, Japan

${ }^{4}$ Japan Science and Technology Agency, PRESTO, 4-1-8 Honcho, Kawaguchi, Saitama 332-0012, Japan

*e-mail: tegi@a-phys.eng.osaka-cu.ac.jp; kim@kuchem.kyoto-u.ac.jp 


\section{Table of Contents}

1. Material preparation and experimental methods

2. Wide-angle $X$-ray diffraction

3. Absorption spectra of CdTe QDs

4. Orbital and geometrical structures of the $\mathrm{Cd}_{26} \mathrm{Te}_{26} \mathrm{QD}$ in the three dimensional periodic superlattice and additional data of band gap energy shifts 


\section{Material preparation and experimental methods}

Chemicals: Tellurium (reagent powder, 99.8\%) was purchased from Sigma. $\mathrm{Cd}\left(\mathrm{ClO}_{4}\right)_{2} \cdot 6 \mathrm{H}_{2} \mathrm{O}$, N-acetyl- $L$-cysteine (NAC), and $\mathrm{NaBH}_{4}$ are from Tokyo kasei. All chemicals are of the highest commercially available purity and used as received without further purification.

Synthesis of CdTe QDs: CdTe QDs were prepared as described in the previous paper [S1]. Briefly, freshly prepared NaHTe solution was injected into the solutions of $\mathrm{Cd}\left(\mathrm{ClO}_{4}\right)_{2} \cdot 6 \mathrm{H}_{2} \mathrm{O}$ and $\mathrm{NAC}$ at $\mathrm{pH}$ 7.0. A molar ratio and concentration of monomers of $\mathrm{Cd}: \mathrm{Te}: \mathrm{NAC}=1.0: 0.2: 1.2$ and $[\mathrm{Cd} 2+]=20 \mathrm{mM}$ were used. Then, $10 \mathrm{~mL}$ of the solution was loaded into an autoclave and incubated at $200{ }^{\circ} \mathrm{C}$ for a specified period.

Layer by layer (LBL) assembly: The substrates of quartz used for the LBL deposition were cleaned by immersion in fresh piranha solution $(1 / 3(\mathrm{v} / \mathrm{v})$ mixture of $30 \% \mathrm{H}_{2} \mathrm{O}_{2}$ and $98 \% \mathrm{H}_{2} \mathrm{SO}_{4}$ ) for 20 min. (Caution: Piranha solution reacts violently with organic materials.) Then, the substrates were rinsed with water, and then used immediately after cleaning. The LBL assembly was performed by sequential dipping of the quartz substrates in aqueous solutions of positively charged poly (diallydimethylammonium) chloride (PDDA, Mw=100,000-200,000) and negatively charged colloidal QDs.

The nano space between the bilayer structure, 
CdTe-QDs/(PDDA/PAA) $/$ /PDDA/CdTe-QDs can be systematically controlled by the spacer $(\mathrm{PDDA} / \mathrm{PAA})_{m}$ composed of positively charged PDDA and negatively charged poly (acryl acid) (PAA), which was deposited by the LBL assembly.

\section{Wide-angle $\mathrm{X}$-ray diffraction}

Wide-angle X-ray diffraction (WAXRD) was also measured on a Rigaku SmartLab with $\mathrm{Cu} \mathrm{K} \alpha$ radiation $(\lambda=0.154 \mathrm{~nm})$. Typical WAXRD pattern for $\mathrm{CdTe}$ QDs, which is shown in Fig.S1, exhibits a diffraction pattern close to that of cubic bulk CdTe. 


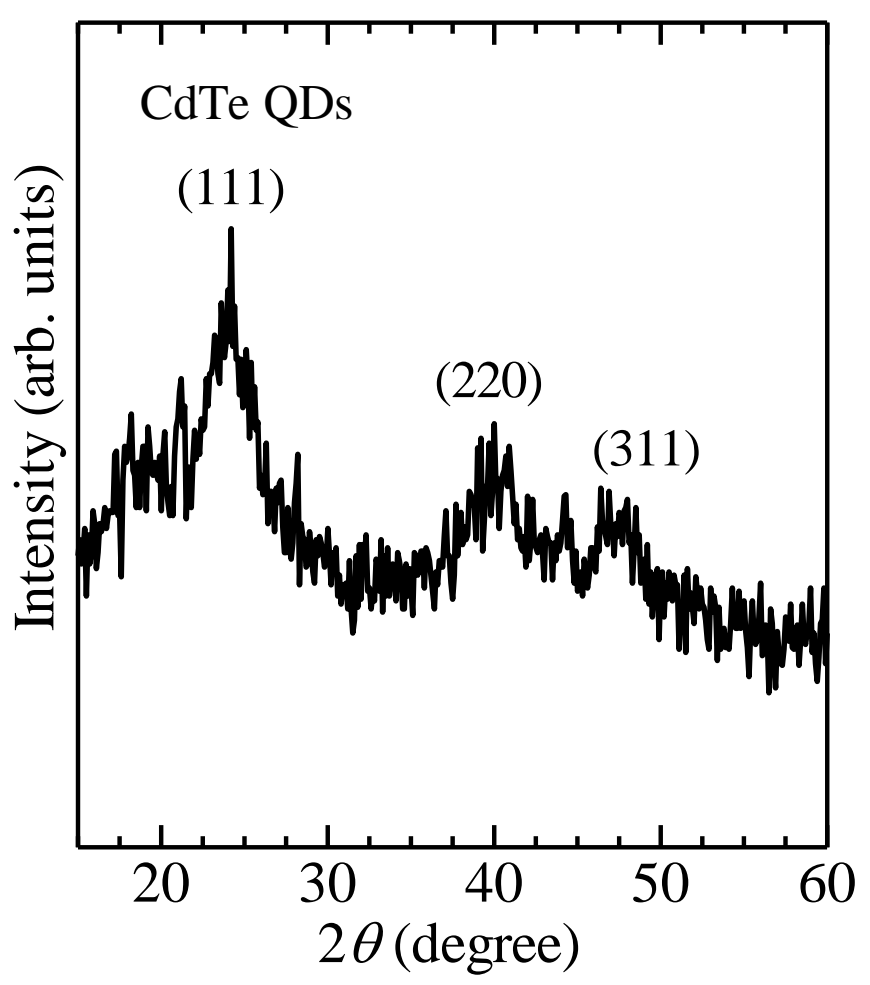

Figure S1. WAXRD pattern of CdTe QDs.

\section{Absorption spectra of CdTe QDs}

Figure S2 shows absorbance spectra of CdTe QDs. The mean diameter of CdTe

QDs were derived from the absorption-peak energy based on the following expression [S3]:

$$
E_{g}(d)=1.596+\frac{1}{0.137 d^{2}+0.206} \text {. }
$$

Here, $d$ denotes diameter of the CdTe QDs. The equation provides a very convenient and accurate way of determining the mean diameter of CdTe QDs from the 
measurements of absorption spectra. The sharp absorption peaks indicate that the size-distribution width of the CdTe QDs is considerably small. The successful preparation of the size-controlled CdTe QDs enabled us to prepare the three dimensional (3D) periodic QD superlattice.

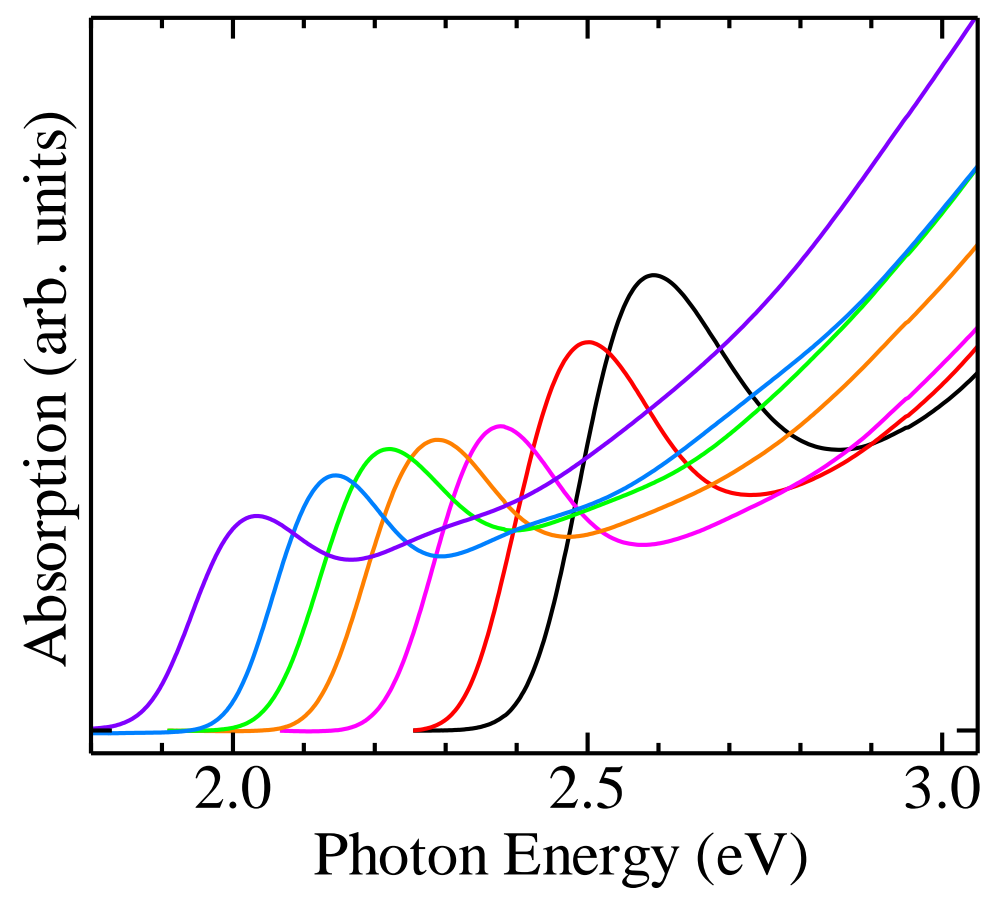

Figure S2. Absorption spectra of CdTe QDs.

4. Orbital and geometrical structures of the $\mathrm{Cd}_{26} \mathrm{Te}_{26} \mathrm{QD}$ in the $3 \mathrm{D}$ periodic superlattice and additional data of band gap energy shifts 

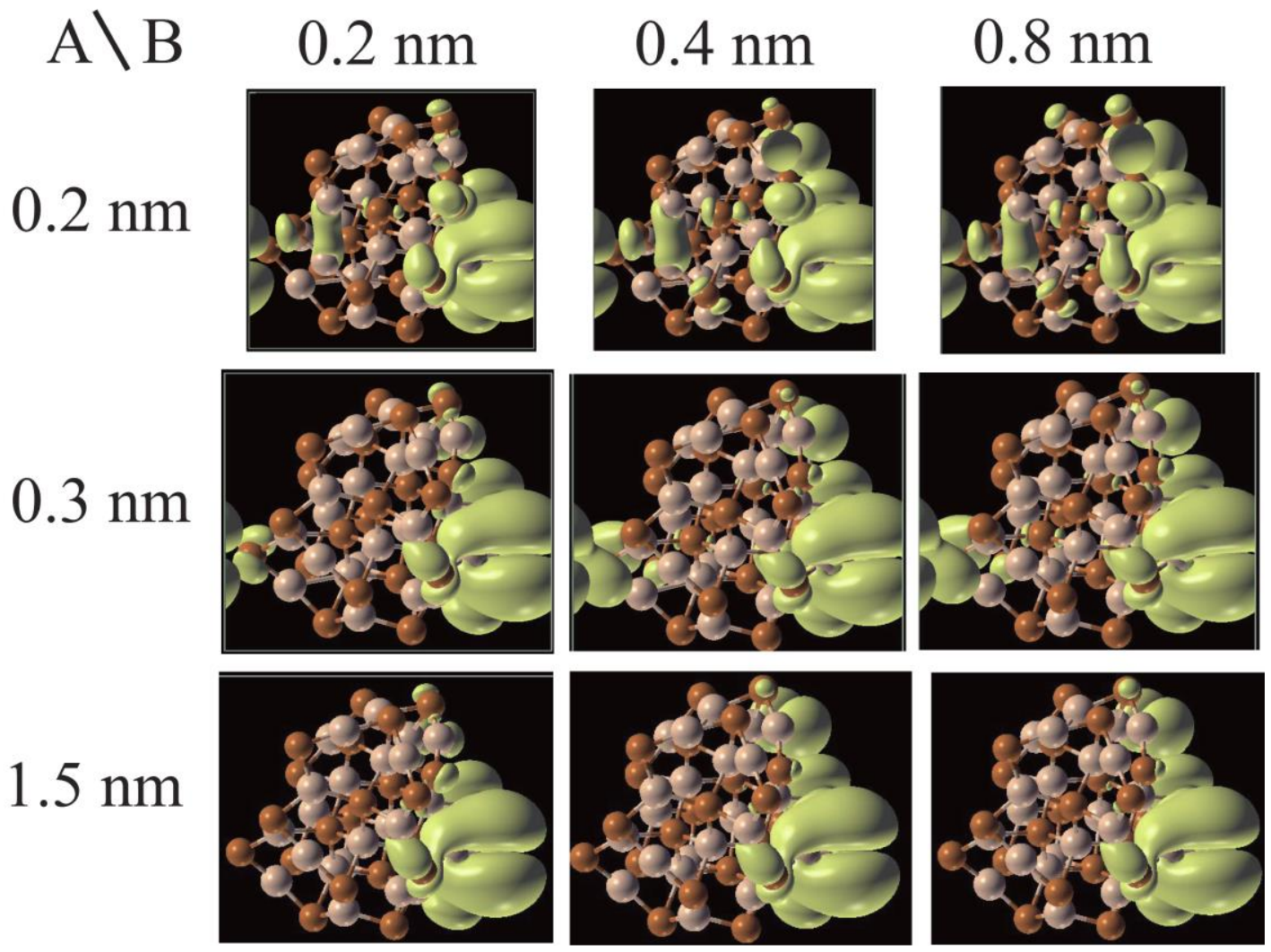

Figure S3: HOMO in the CdTe QD superlattice for various A and B. The HOMO is delocalized over the neighboring $\mathrm{Cd}_{26} \mathrm{Te}_{26}$ QDs mainly in the xy-plane with the small nano space. The HOMO becomes localized onto each $\mathrm{Cd}_{26} \mathrm{Te}_{26} \mathrm{QD}$ as the nano space (A, A, B) becomes larger. There is little HOMO delocalization along z-axis. This asymmetric delocalization is due to the effect of the surface defects enhanced by the strong Coulomb interaction between positive $\mathrm{Cd}$ and negative $\mathrm{Te}$ atoms. Taking into account the fact that the HOMO delocalization is only partial even in the xy-plane, the current quantum resonance is mainly caused by the delocalization of the LUMO drawn in Fig.4c. 

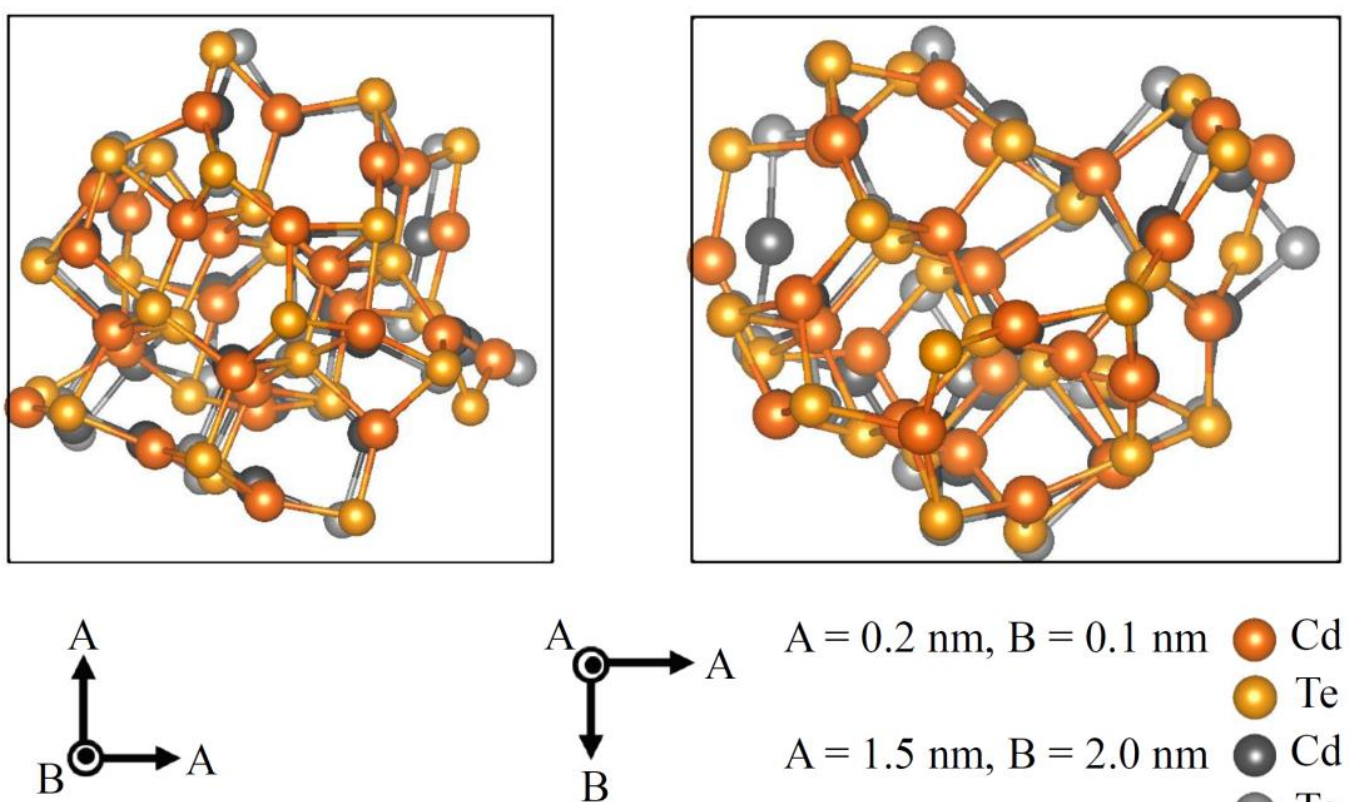

$$
\begin{array}{r}
\mathrm{A}=0.2 \mathrm{~nm}, \mathrm{~B}=0.1 \mathrm{~nm} \odot \mathrm{Cd} \\
\mathrm{A}=1.5 \mathrm{~nm}, \mathrm{~B}=2.0 \mathrm{~nm} \\
\odot \mathrm{Cd} \\
\odot \mathrm{Te}
\end{array}
$$

Figure S4: Different views of $\mathrm{Cd}_{26} \mathrm{Te}_{26}$ QD structures in the 3D QD superlattice at (A, A, $B)=(0.2 \mathrm{~nm}, 0.2 \mathrm{~nm}, 0.1 \mathrm{~nm})$ and $(1.5 \mathrm{~nm}, 1.5 \mathrm{~nm}, 2.0 \mathrm{~nm})$. The structural deformation is significant at the small nano space due to the compression by the surrounding QDs. 
a

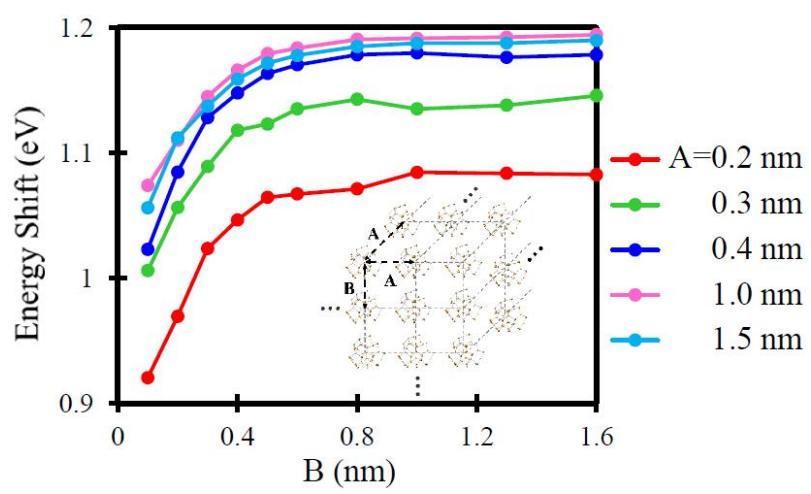

b

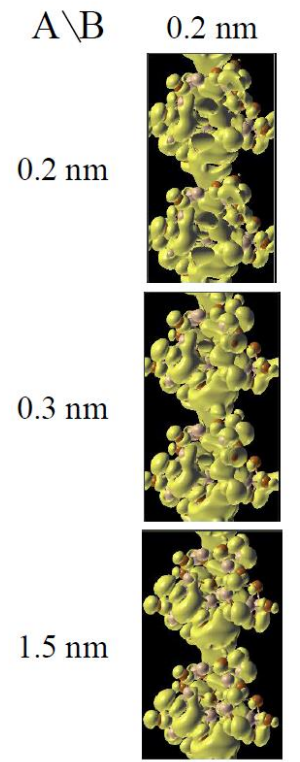

$0.4 \mathrm{~nm}$

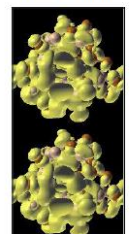

$0.8 \mathrm{~nm}$
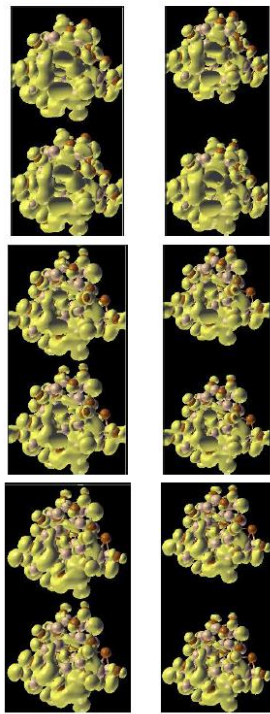

Figure S5: (a) Same as Fig. 4a but for a CdTe QD superlattice with a unit cell composed of a QD dimer, that is, two $\mathrm{Cd}_{26} \mathrm{Te}_{26}$ QDs. All shifts of HOMO-LUMO band gap energies of the CdTe QD superlattice exhibit the similar results to Fig.4a. (b) Same as Fig. 4b but for the CdTe QD superlattice with a unit cell composed of a $\mathrm{Cd}_{26} \mathrm{Te}_{26}$ QD dimer. All the delocalized LUMO of the CdTe QD superlattice is similar to the LUMO shown in Fig.4b. In the case of the QD superlattice with the QD dimer, the two $\mathrm{Cd}_{26} \mathrm{Te}_{26}$ QDs in the unit cell are equally compressed; the QD diameters along the xyz-directions are $(1.39 \mathrm{~nm}, 1.38 \mathrm{~nm}, 1.17 \mathrm{~nm})$ and $(1.40 \mathrm{~nm}, 1.40$ $\mathrm{nm}, 1.24 \mathrm{~nm})$ for the nano space set as $(A, A, B)=(0.2 \mathrm{~nm}, 0.2 \mathrm{~nm}, 0.1 \mathrm{~nm})$ and $(1.5 \mathrm{~nm}, 1.5 \mathrm{~nm}, 2.0$ $\mathrm{nm})$, respectively, which are almost identical with the QD diameters obtained with the single $\mathrm{Cd}_{26} \mathrm{Te}_{26} \mathrm{QD}$ unit cell. All of these data clearly demonstrate that similar repulsive and attractive forces between neighboring QDs exist regardless of the unit cells. 


\begin{tabular}{|c|c|c|c|c|c|c|}
\hline \multirow{2}{*}{\multicolumn{2}{|c|}{$\mathrm{E}_{\mathrm{g}}(\mathrm{eV})$}} & \multicolumn{5}{|l|}{$\mathbf{A}(\mathrm{nm})$} \\
\hline & & \multirow{2}{*}{$\frac{0.2}{0.9130}$} & \multirow{2}{*}{$\frac{0.3}{0.9956}$} & \multirow{2}{*}{$\frac{0.4}{1.0287}$} & \multirow{2}{*}{$\frac{1.0}{1.0487}$} & \multirow{2}{*}{$\frac{1.5}{1.0381}$} \\
\hline B & 0.1 & & & & & \\
\hline$(\mathrm{nm})$ & 0.2 & 0.9709 & 1.0438 & 1.0834 & 1.0981 & 1.0913 \\
\hline & 0.3 & 1.0122 & 1.0874 & 1.1253 & 1.1340 & 1.1382 \\
\hline & 0.4 & 1.0619 & 1.1214 & 1.1461 & 1.1623 & 1.1657 \\
\hline & 0.5 & 1.0731 & 1.1319 & 1.1586 & 1.1750 & 1.1702 \\
\hline & 0.6 & 1.0740 & 1.1391 & 1.1653 & 1.1808 & 1.1764 \\
\hline & 0.8 & 1.0768 & 1.1430 & 1.1704 & 1.1864 & 1.1824 \\
\hline & 1.0 & 1.0864 & 1.1445 & 1.1719 & 1.1882 & 1.1846 \\
\hline & 1.3 & 1.0822 & 1.1441 & 1.1712 & 1.1881 & 1.1913 \\
\hline & 1.6 & 1.0814 & 1.1450 & 1.1715 & 1.1892 & 1.1855 \\
\hline
\end{tabular}

Table S1: Numerical data of band-gap energies of the CdTe QD superlattice for $(A, A, B)$ shown in Fig.4a. The band-gap energy does not saturate before $(A, A, B)=(1.0 \mathrm{~nm}, 1.0 \mathrm{~nm}, 1.0 \mathrm{~nm})$. 


\section{References}

(1) Bu, H., Kikunaga, H., Shimura, K., Takahashi, K., Taniguchi, T., \& Kim, D. Hydrothermal synthesis of thiol-capped CdTe nanoparticles and their optical properties, Phys. Chem. Chem. Phys. 15, 2903-2911 (2013).

(2) Kim, D., Okahara, S., Nakayama, M., \& Shim, Y. Experimental verification of Förster energy transfer between semiconductor quantum dots, Phys. Rev. B 78, 153301 (2008).

(3) Donega, C. M. \& Koole, K. Size dependence of the spontaneous emission rate and absorption cross section of CdSe and CdTe quantum dots, J. Phys. Chem. C 113, 6511-6520 (2009). 